\title{
Evaluation of physical growth and sexual maturation rating of Adolescent boys
}

\author{
Gedam DS', Waseem $\mathbf{M}^{2}$, Chansoria $\mathbf{M}^{3}$ \\ 'Dr. D Sharad Gedam, MBBS, MD, Associate Professor, LN Medical College, Bhopal, India, ${ }^{2}$ Dr. Md Waseem, MBBS, MD, \\ Assistant Professor, RD Gardi Medical College, Ujjain, India, ${ }^{3}$ Dr. Maya Chansoria, MBBS, MD, Professor and HOD, Netaji \\ Subhas Chandra Bose Medical College, Jabalpur, India.
}

Address for correspondence: Dr. D Sharad Gedam, E-mail: sharad_gedam@rediffmail.com

\begin{abstract}
Introduction: The present study is an attempt to evaluate the incidence of malnutrition as well as obesity. We have evaluated physical growth and sexual maturity rating of 10-18 years adolescent boys-overweight Vs Normal Vs Underweight. Material and Methods: A random sample of 530 adolescent boys (one private English Medium and one Govt. Hindi Medium School) between the ages of 10-18 years during the period of August 2008 to August 2009 constituted the material for this study. Weight, Height and BMI and Sexual maturation rating (SMR) were calculated. Results: Nearly 39\% of the adolescent boys studied were either underweight (31\%) or overweight (8\%). All overweight adolescent boys have their mean weight more as compared to $\mathrm{WHO} 50^{\text {th }}$ centile. Normal weight and underweight adolescent boys have lesser mean weight as compared to $\mathrm{WHO} 50^{\text {th }}$ centile. All overweight and normal weight adolescent boys have their mean height lesser as compared to WHO $50^{\text {th }}$ centile. All underweight adolescent boys except for age group of $12 \mathrm{yr}$ have their mean height lesser as compared to WHO $50^{\text {th }}$ centile. Underweight boys achieved different stages of genital growth prior to normal weight and overweight boys but lag behind as compared to Tanner. It was obvious that underweight boys achieved different stages of pubic hair growth prior to normal weight and overweight boys. Conclusion: Present study indicates that incidence of overweight children is also increasing significantly. Adolescent psychological need should be assessed and they should be given knowledge regarding their normal sexual growth pattern to decrease their anxiety.
\end{abstract}

Key words: Obesity in Adolescent, SMR, Early maturer, BMI in Adolescent.

\section{Introduction}

$\mathrm{T}$ he origin of the word adolescence is from Greek Latin word 'adolesere' which means 'to grow' or to grow to maturity'. It is a period of rapid and extensive series of anatomical, biochemical, emotional, psychosocial and secondary sexual changes in encompassing the age range from $10-19$ years. The pattern of these changes is in turn modified and individualized by genetic potential as well as environmental and socio-cultural influences.

Today adolescents are going through stormy period of life with tremendous academic, emotional pressures and expectations because of changing lifestyle, higher

Manuscript received: $14^{\text {th }}$ Dec 2011

Reviewed: $17^{\text {th }}$ March 2012

Author Corrected: $2^{\text {nd }}$ April 2012

Accepted for Publication: $30^{\text {th }}$ May 2012 degree of competitiveness, peer pressure and influences of tube culture.

Adolescence proceeds across three distinct periodsearly, middle and late, each marked by a characteristic set of salient biologic, psychological and social issues. However individual variation is substantial, both in terms of the timing of somatic and secondary sexual changes and quality of the adolescents experience.

Growth assessment is an essential component of paediatric health surveillance because almost any problem within the physiologic, interpersonal and social domains can adversely affect growth.

\section{Material and Methods}

A random sample of 530 adolescent boys between the ages of 10-18 years during the period of August 2008 
to August 2009 constituted the material for this study. A random sample was drawn from two different schools; one private English Medium and one Govt. Hindi Medium School reflecting students from all socioeconomic strata of society.

A formal request was made to the Head of these institutions for permission to carry out this study. Having ensured our acceptance, the students were approached in small batches of 40 students at a time in the absence of class teachers. They were requested to fill in a questionnaire after being explained that the object of the study was to determine their physical growth and sexual maturation. They were examined for genital and pubic hair for their sexual maturation rating confidentially.

Protection of individual identity and confidentiality of responses were ensured by maintaining anonymity by allotting code numbers to each subject. To avoid any deliberate distortion of responses they were promised that the result would be communicated to them after analysis.

Section l: of questionnaire was designed to gather general information regarding age, sex, birth order, parental education, occupation, per capita income, Weight, Height and BMI.

\section{a) Weight:}

The weighing scale used in this survey measuring in kilo grams up to accuracy of $100 \mathrm{gms}$. The zero error of the machine was regularly eliminated before taking the weight. Boys were made to stand barefoot on the centre of the platform, without touching anything and their weight recorded.

\section{b) Height:}

The height was measured with an anthrop meter. It consisted of wooden platform $24^{\prime \prime} \mathrm{X} 18^{\prime \prime}$ to which $10^{\prime \prime}$ wide vertical plank was fixed, which could measure up to a tenth of a centimetre. A rectangular wooden head board was used. Boys with barefooted, were made to stand on the flat platform with the feet joined and making an angle of $45^{\circ}$ with each other each other. Heels, buttocks, shoulders and back of head were made to touch the upright plank. The head was held comfortable erect, such that the lower borders of the orbits were in the some horizontal plane is the external auditory meatus. The hands were made to hang at the sides in a natural manner. During the measurement, the child was asked to take a deep breath, relax the shoulders and stretch up to be as tall as possible. Top knots of Sikh boys were opened. The headpiece was moved down until it touched the child's head and keeping the headpiece in position the child was asked to get off the anthrop meter. The height of the child was then recorded.

\section{C) Body Mass Index (BMI)}

It is calculated by formula given below $\mathrm{BMI}=$ Weight/ Height $X$ Height

Weight in Kilogram \& Height in meters.

Body Mass Index (BMI) Classification of children and adolescent

\begin{tabular}{|l|l|}
\hline BMI Percentile for age & \multicolumn{1}{c|}{ Weight status } \\
\hline$<5^{\text {th }}$ percentile & Underweight \\
\hline $5^{\text {th }}-84^{\text {th }}$ percentile & Normal weight \\
\hline $85^{\text {th }}-94^{\text {th }}$ percentile & At risk for overweight \\
\hline$\geq 95^{\text {th }}$ percentile & Overweight \\
\hline
\end{tabular}

Overweight was defined as BMI $95^{\text {th }}$ percentile or more ${ }^{2}$.

Underweight was defined as $\mathrm{BMI}$ less then $5^{\text {th }}$ percentile 2 (NCHS 2000).

Section II: Sexual maturation rating (SMR) was calculated by physical examination (Genital stage and Pubic hair stage) $)^{3}$.

\section{GENITAL GROWTH: ${ }^{3}$}

Stage I: Pre-adolescent, testes, scrotum and penis of about the same size and proportions as in early childhood.

Stage II: Enlargement of scrotum and of testes. The skin of the scrotum reddens and changes in texture little or no enlargement of penis.

Stage III: Enlargement of the penis, that occurs at first mainly in length. Further growth of testes and scrotum.

Stage IV: Increase size of penis with growth in breadth and development of glans. Further enlargement of testes and scrotum; increased darkening of scrotal skin.

Stage V: Genitalia adult in size and shape. No further enlargement takes place.

\section{Pubic HAIR}

Stage I: Pre-adolescent. The vellus over the pubes is not further developed than that over abdominal wall, i.e. no pubic hair. 
Stage II: Sparse growth of long, slightly pigmented downy hair, straight to only slightly curled, appearing chiefly at the base of the penis.

Stage III: Considerably darker, coarser and more curled. The hairs spread sparsely over the junction of the pubes. It is at this stage that pubic hair is first seen in an ordinary black and white photograph of entire body.

Stage IV: Hair now resembles adult in type, but area covered by it is still considerably smaller than in adult. No spread to the medial surface of thigh.

Stage V: Adult quantity and quality with distribution of the horizontal pattern and spread to medial surface of things but not up linea alba on above the base of the inverse triangle.

\section{Results}

Table 1: Showing percentage wise distribution of overweight, normal weight and underweight adolescent boys

\begin{tabular}{|l|r|r|}
\hline & \multicolumn{1}{|c|}{ (N) } & Percentage (\%) \\
\hline Overweight & 42 & 8 \\
\hline Normal weight & 324 & 61 \\
\hline Underweight & 164 & 31 \\
\hline & $\mathbf{5 3 0}$ & $\mathbf{1 0 0}$ \\
\hline
\end{tabular}

All overweight adolescent boys had their mean weight more as compared to WHO $50^{\text {th }}$ centile. Normal weight and underweight adolescent boys has lesser mean weight as compared to $\mathrm{WHO} 50^{\text {th }}$ centile. All overweight and normal weight adolescent boys had their mean height lesser as compared to $\mathrm{WHO} 50^{\text {th }}$ centile. All underweight adolescent boys except for age group of $12 \mathrm{yr}$ had their mean height lesser as compared to WHO $50^{\text {th }}$ centile.

As per Table 5 maximum number of late adolescent belongs to $\mathrm{G} 3$ to $\mathrm{G} 5$ category.

Nearly one third of the subjects studied deviated from the group of normal maturity. They were either late maturer (29.4\%) or early maturer (4\%).

From the present study it was obvious that underweight boys achieved different stages of genital growth prior to normal weight and overweight boys but lag behind as compared to Tanner².

It was obvious that underweight boys achieved different stages of pubic hair growth prior to normal weight and overweight boys. It was also obvious that pubic hair growth stage 2 and stage 3 for underweight boys precedes Tanner stage.

As per Table 1 Nearly 39\% of the adolescent boys studied were either underweight $(31 \%)$ or overweight (8\%) remaining $61 \%$ were having normal weight.

Table 2: Age wise distribution of overweight, normal weight and underweight adolescent boys showing age wise distribution of boys. Incidence of underweight adolescent is maximum in late adolescent while overweight adolescent belongs to age group 15-17 years.

\begin{tabular}{|c|c|c|c|c|}
\hline Age(Yrs) & Total & Overweight & Normal weight & Underweight \\
\hline & $\mathbf{5 3 0}$ & $\mathbf{4 2}$ & $\mathbf{3 2 4}$ & $\mathbf{1 6 4}$ \\
\hline \multirow{2}{*}{11} & 30 & 3 & 18 & 9 \\
& $5.67 \%$ & $7.14 \%$ & $5.56 \%$ & 22 \\
\hline \multirow{2}{*}{12} & 36 & 3 & $6.79 \%$ & 11 \\
& $6.79 \%$ & $7.14 \%$ & 16 & 16 \\
\hline \multirow{2}{*}{13} & 36 & 4 & $4.49 \%$ & $9.76 \%$ \\
\hline \multirow{2}{*}{14} & $6.79 \%$ & $9.52 \%$ & 52 & 24 \\
& 80 & 4 & $16.05 \%$ & $14.63 \%$ \\
\hline \multirow{2}{*}{15} & $15.09 \%$ & $9.52 \%$ & 48 & 20 \\
& 80 & 12 & $14.81 \%$ & $12.2 \%$ \\
\hline \multirow{2}{*}{16} & $15.09 \%$ & $28.57 \%$ & 88 & 56 \\
& 148 & 4 & $27.16 \%$ & $34.14 \%$ \\
\hline \multirow{2}{*}{17} & $27.92 \%$ & $9.52 \%$ & 80 & 28 \\
\hline (N) & 120 & 12 & $24.69 \%$ & $17.07 \%$ \\
\hline
\end{tabular}


Evaluation of physical growth and sexual maturation rating of Adolescent boys

Table 3: Showing mean weight $(\mathrm{kg})$ in overweight, normal weight and underweight adolescent boys (present study) as compared to WHO.

\begin{tabular}{|c|c|c|c|c|}
\hline Age & $\begin{array}{c}\text { WHO } \\
\mathbf{5 0}^{\mathbf{T H}} \text { centile }\end{array}$ & Overweight & Normal weight & Underweight \\
\hline 11 & 31.7 & $42.7 \pm 0.6$ & $30.6 \pm 2.1$ & $27.9 \pm 1.6$ \\
\hline 12 & 40.3 & $48.3 \pm 1.5$ & $39.2 \pm 3.24$ & $32.5 \pm 2.9$ \\
\hline 13 & 45.4 & $52 \pm 3.37$ & $43.3 \pm 1.44$ & $33.5 \pm 3.5$ \\
\hline 14 & 50.8 & $57.3 \pm 2.40$ & $44.7 \pm 7.7$ & $39.2 \pm 5.8$ \\
\hline 15 & 56 & $61 \pm 1.15$ & $50.7 \pm 5.9$ & $44.8 \pm 5.3$ \\
\hline 16 & 60.8 & $63.6 \pm 3.9$ & $49.5 \pm 5.7$ & $45.0 \pm 4.1$ \\
\hline 17 & 64.4 & $65.5 \pm 4.03$ & $51.8 \pm 4.8$ & $46.6 \pm 8.4$ \\
\hline
\end{tabular}

Table 4: Showing mean height $(\mathrm{cm})$ in overweight, normal weight and underweight adolescent boys (present study) as compared to WHO.

\begin{tabular}{|c|c|c|c|c|}
\hline Age & $\begin{array}{c}\text { WHO } \\
\mathbf{5 0}^{\text {th }} \text { Centile }\end{array}$ & Overweight & Normal weight & Underweight \\
\hline 11 & 143.3 & $131 \pm 1.0$ & $133 \pm 2.0$ & $142.8 \pm 3.9$ \\
\hline 12 & 149 & $140.3 \pm 2.0$ & $144.6 \pm 7.51$ & $150.2 \pm 5.2$ \\
\hline 13 & 155.7 & $144 \pm 1.41$ & $152.9 \pm 4.29$ & $152.3 \pm 8.32$ \\
\hline 14 & 163.5 & $152.8 \pm 2.6$ & $160.4 \pm 13.5$ & $155.6 \pm 10.7$ \\
\hline 15 & 169.8 & $157.6 \pm 2.08$ & $164.5 \pm 6.75$ & $163.2 \pm 5.8$ \\
\hline 16 & 173.5 & $160.1 \pm 4.03$ & $165.8 \pm 8.65$ & $170.0 \pm 8.5$ \\
\hline 17 & 175.3 & $161.7 \pm 5.56$ & $168.1 \pm 7.81$ & $170.4 \pm 9.0$ \\
\hline
\end{tabular}

Table 5: Showing percentage wise distribution of different stages of genital growth in adolescent boys

\begin{tabular}{|c|c|c|c|c|c|c|c|c|}
\hline Age Stage & $\mathbf{1 1}$ & $\mathbf{1 2}$ & $\mathbf{1 3}$ & $\mathbf{1 4}$ & $\mathbf{1 5}$ & $\mathbf{1 6}$ & $\mathbf{1 7}$ & Total \\
\hline G1 & 7 & 3 & 1 & 0 & 0 & 0 & 0 & $11(2 \%)$ \\
\hline G2 & 17 & 21 & 15 & 20 & 4 & 1 & 0 & $78(14.8 \%)$ \\
\hline G3 & 6 & 11 & 17 & 50 & 29 & 35 & 1 & $149(28.1 \%)$ \\
\hline G4 & 0 & 1 & 3 & 9 & 36 & 32 & 6 & $87(16.4 \%)$ \\
\hline G5 & 0 & 0 & 0 & 1 & 11 & 80 & 113 & $205(38.7 \%)$ \\
\hline Total & $\mathbf{3 0}$ & $\mathbf{3 6}$ & $\mathbf{3 6}$ & $\mathbf{8 0}$ & $\mathbf{8 0}$ & $\mathbf{1 4 8}$ & $\mathbf{1 2 0}$ & $\mathbf{5 3 0}$ \\
\hline
\end{tabular}

Table 6: Showing percentage wise distribution of Early maturer, Normal maturer and Late maturer

\begin{tabular}{|l|c|c|}
\hline & (N) & Percentage \\
\hline Early maturer & 21 & $4 \%$ \\
\hline Normal maturer & 353 & $66.6 \%$ \\
\hline Late maturer Total & 156 & $29.4 \%$ \\
\hline \multicolumn{2}{|c|}{$\quad \mathbf{5 3 0}$} & $\mathbf{1 0 0 \%}$ \\
\hline
\end{tabular}

Table 7: Showing the mean ages at which different stages of genital growth are achieved as compared with Tanner is shown

\begin{tabular}{|l|c|c|c|c|}
\hline Source & G2 & G3 & G4 & G5 \\
\hline 1.Present study & & & & $15.3 \pm 0.95$ \\
Overweight & $13.4 \pm 1.14$ & $15.0 \pm 1.04$ & $15.4 \pm 0.72$ & $16.6 \pm 0.56$ \\
Normal weight & $12.8 \pm 1.28$ & $14.5 \pm 1.32$ & $14.8 \pm 1.14$ & $16.3 \pm 0.63$ \\
Underweight & $12.3 \pm 1.25$ & $13.6 \pm 1.33$ & 13.8 & 14.9 \\
\hline 2. Marshall and Tanner & 11.6 & 12.9 & & \\
\hline
\end{tabular}


Table 8: Showing the mean ages at which different stages of pubic hair growth are achieved as compared with Tanner is shown

\begin{tabular}{|l|c|c|c|c|}
\hline \multicolumn{1}{|c|}{ Source } & P 2 & P 3 & P 4 & P 5 \\
\hline 1. Present study & & & & $16.9 \pm 0.8$ \\
Overweight & $13.5 \pm 1.3$ & $14.6 \pm 1.02$ & $15.6 \pm 1.12$ & $16.6 \pm 0.61$ \\
Normal weight & $12.6 \pm 1.2$ & $14.5 \pm 1.3$ & $15.4 \pm 0.82$ & $16.3 \pm 0.59$ \\
Underweight & $12.5 \pm 1.28$ & $13.8 \pm 1.35$ & $14.8 \pm 1.08$ & 15.1 \\
\hline 2. Marshall and Tanner & 13.4 & 13.9 & 14.4 & \\
\hline
\end{tabular}

\section{Discussion}

\section{Physical growth}

As per Table 1 Present study shows the prevalence of overweight and underweight adolescent boys is $8 \%$ and $31 \%$ respectively.

Prevalence of overweight increased in Brazil (from 4.1 to 13.9), China (from 6.4 to 7.7) and United states (from 15.4 to 25.6) whereas decreased in Russia (from 14.5 to 13.1). Prevalence of underweight increased in Russia (from 6.9 to 8.1) but decreased in Brazil (from 14.8 to 8.6 ) \& China (from 14.5 to 13.1$)^{4}$.

Sexual maturation in normal weight adolescent boys achieved prior to both overweight and underweight adolescent boys but underweight adolescent boys mature sexually before overweight adolescent boys ${ }^{4}$.

Prevalence of overweight and underweight is $11 \%$ and $10 \%$ respectively in our country ${ }^{5}$.

Overweight and underweight adolescents predispose more illness, both physical and mental \& more personality changes in comparison to normal weight adolescents. Overweight causes more morbidity and mortality than underweight. Overweight not only causes much psychological morbidity, but is also a primary risk factor in development of hypertension, cardiovascular disease, stroke, diabetes mellitus, hyperlipidemia, osteoarthritis and cancer of prostate ${ }^{6}$. Underweight causes fatigue, lack of energy and increased susceptible to infection ${ }^{6}$. These adolescents are more risk for hip fracture ${ }^{6}$. Epidemiological evidence indicates that overweight as well as underweight have a higher prevalence of psychiatric disorders ${ }^{7}$.

Overweight groups have tendency to be sociable, warm and cheerful. They are also more impulsive \& curious.

Underweight groups have more anxiety, anger and depression ${ }^{8}$.
As per Table 3, All overweight adolescent boys have their mean weight more as compared to $\mathrm{WHO} 50^{\text {th }}$ centile. Normal weight and underweight adolescent boys have lesser mean weight as compared to $\mathrm{WHO} 50^{\text {th }}$ centile. All overweight adolescent boys of present study have their mean weight more as compared to studies done on average Indian adolescent boys 9,10,11,12 $^{\text {. Underweight }}$ adolescent boys of present study have their mean weight lesser as compared to western and average Indian adolescent boys ${ }^{3}$. As per Table 4 All overweight and normal weight adolescent boys had their mean height lesser as compared to $\mathrm{WHO} 50^{\text {th }}$ centile. All underweight adolescent boys except for age group of $12 \mathrm{yr}$ had their mean height lesser as compared to $\mathrm{WHO} 50^{\text {th }}$ centile.

It is well established that the time of maximum growth in height is closely correlated with sexual maturation $13,14,15,16,17$. All underweight adolescent boys of present study had their mean height more as compared to studies done on average Indian adolescent boys 9 ,10,11,12. Overweight adolescent boys of present study have their mean height lesser as compared to western and average Indian adolescent boys ${ }^{3}$.

As per Table $6 \& 7$ : From the present study it was obvious that underweight boys achieved different stages of genital growth prior to normal weight and overweight boys but lag behind as compared to Tanner ${ }^{3}$.

Similar results are seen in this study where Sexual maturation in normal weight adolescent boys achieved prior to both overweight and underweight adolescent boys but underweight adolescent boys mature sexually before overweight adolescent boys 4 .

Studies done by ${ }^{4,18}$ Chicago revealed early maturing boys had lower BMI than their counterparts; whereas early maturing girls had greater measures of BMI. Early sexual maturation was related to increased height but not to increased body weight in boys, while it was associated with both increased height and weight in girls. 
As per Table 8 it was obvious that underweight boys achieved different stages of pubic hair growth prior to normal weight and overweight boys. It was also obvious that pubic hair growth stage 2 and stage 3 for underweight boys precedes Tanner stage.

Frisch and Revelle ${ }^{19}$ offered a hypothesis that attainment of critical body size might be a factor involved in the alteration of the sensitivity of the hypothalamic centres responsible for the onset of puberty. They are also of the opinion that a critical body size is attained before final stages of maturity are achieved.

Accurate maturity assessment of boys should include not only clinical examination but also radiological assessment of age and estimate of hormones secretion.

The various stages of adolescent begin at different ages in each individual. Hence, longitudinal studies are indispensable for correct assessment.

\section{Conclusion}

Underweight adolescent boys achieved different stages of sexual maturation prior to normal weight and overweight adolescent boys. All overweight adolescent boys of present study had their mean weight more as compared to WHO $50^{\text {th }}$ centile. Normal weight and underweight adolescent boys had lesser mean weight as compared to WHO $50^{\text {th }}$ centile. All overweight and normal weight adolescent boys had their mean height lesser as compared to WHO $50^{\text {th }}$ centile. All underweight adolescent boys except the age group of $12 \mathrm{yr}$ had their mean height lesser as compared to WHO $50^{\text {th }}$ centile. Overweight adolescent boys constituted $8 \%$, normal weight $61 \%$ and underweight adolescent boys $31 \%$. Early maturing adolescent boys constitutes $4 \%$, normal maturing $66.6 \%$ and late maturing adolescent boys $29.4 \%$. Further longitudinal studies, correlating anthropometry, bone age and secondary sex characters with hormonal secretion need to be undertaken.

Acknowledgements: The authors are thankful to Dr. Maya Chansoria MD Prof. and Head of Department of Paediatrics, Netaji Subhash Chandra Bose Medical College, Jabalpur, India for her guidance, supervision and encouragement in conducting this study. We also want to thanks all our young subjects, their parents \& school authority for permission to conduct this study.

\footnotetext{
Funding: Nil

Conflict of Interest: None

Permission from IRB: Yes
}

\section{References}

1. Agarwal K, Satyavathi NK. Review on adolescent growth studies part A: Physiological aspect and environmental factors. Indian J Pediatr 2004;71(6):565-67

2. Kliegman, Behrman, Jenson. Nelson Text Book of Pediatrics, $17^{\text {th }}$ ed. W. B. Saunders Company; 2004.p 61.

3. Tanner JM. Growth at Adolescence, Oxford $2^{\text {nd }}$ ed. Oxford, England, Blackwell scientific publications; 1962.

4. Wang Y, Monteiro C, Popkin M. Trends of obesity and underweight in older children and adolescents in the United States, Brazil, China, and Russia. Am J Clin Nutr 2002;75(6):971-77.

5. Elizabeth KE. A Novel growth assessment chart for adolescents. Indian Pediatr 2001;38:1061-64.

6. Elizabeth KE. Nutrition and child development, $3^{\text {rd }}$ ed. Paras publication, Hyderabad;2004:p 61.

7. Lusky A, Barell V, Lubin F, et al. Relationship between morbidity and extreme values of body mass index in adolescents. Int J Epidemiol 1996;25(4):82934.

8. Terracciano A, Sutin R, McCrae R, et al. Psychosom Med 2009;71 ( 6):682-89.

9. Rao. Health of school children in Pune. Indian J Child Health 1957;6:877.

10. Singh R. A cross-sectional study of growth in five somatometric of Punjabi boys aged 11-18 yrs. Am J Phys Anthropol 1970;32:129.

11. Agarwal DK, Agarwal KN, Upadhyay S, et al. J,Physical and Sexual growth pattern of affluent Children 5-18 years Age. Indian Paediatr 1992;29(10):1248-82.

12. Tripathi AM Physical growth during adolescence in Delhi School children. Indian Paediatr 1976;13:191.

13. Boas $F$. The growth of first-born children. Sci Am 1895;1:402-4.

14. Simmons K, Greulich W.Menarchial Age and the Height, Weight and Skeletal Age of Girls 7-17 years. J Pediatr 1943;22:518-48.

15. Stuart HC. Normal Growth and Development during Adolescence. N Engl J Med 1946;234:666-72. 
16. Nicolson AB, Hanley C. Indices of physiological maturity: derivation and interrelationships. Child Dev 1953;24(1):3-38.

17. Deming J. Application of the Gompertz curve to the observed pattern of growth in length of 48 individual boys and girls during the adolescent cycle of growth. Hum Biol 1957;29:83-120.
18. Wang Y. Is obesity associated with early sexual maturation? A comparison of the association in American boys verse girls. Pediatrics 2002;110:903-10.

19. Frisch R, Revelle R. The height and weight of girls and boys at the time of initiation of the adolescent growth spurt and relationship to menarche. Hum Biol 1971; 43: 140-159.

\section{How to cite this article?}

Gedam DS, Waseem M, Chansoria M. Evaluation of physical growth and sexual maturation rating of Adolescent boys. J Nepal Paediatr Soc 2012;32(2):117-123. 